Research Article

\title{
Microbiological Isolates and Antibiotic Susceptibilities in Cases of Posttraumatic Endophthalmitis: A 15-Year Review
}

\author{
Chunhong Liu, ${ }^{1,2}$ Jian Ji, ${ }^{1}$ Zhujian Wang, ${ }^{1}$ Huiwen Chen, ${ }^{1}$ Wenjun Cao $\mathbb{D}^{1,2}$ \\ and Xinghuai Sun ${ }^{2}$ \\ ${ }^{1}$ Department of Clinical Laboratory, Eye and ENT Hospital, Shanghai Medical College, Fudan University, Shanghai, China \\ ${ }^{2}$ Department of Ophthalmology and Visual Science, Eye and ENT Hospital, Shanghai Medical College, Fudan University, \\ Shanghai, China
}

Correspondence should be addressed to Wenjun Cao; wgkjyk@aliyun.com

Received 9 September 2019; Revised 15 November 2019; Accepted 7 December 2019; Published 29 April 2020

Academic Editor: Enrique Mencía-Gutiérrez

Copyright ( $\odot 2020$ Chunhong Liu et al. This is an open access article distributed under the Creative Commons Attribution License, which permits unrestricted use, distribution, and reproduction in any medium, provided the original work is properly cited.

\begin{abstract}
Purpose. The objective of this study was to evaluate the microbiological spectrum and antibiotic susceptibilities of isolates in posttraumatic endophthalmitis over a 15-year period. Methods. A retrospective study of 3,163 posttraumatic endophthalmitis cases was conducted between July 2004 and July 2019. The outcome measures included the microbiological spectrum and antibiotic susceptibilities. Chi-squared tests were conducted to detect trends in changes in antibiotic sensitivity over the 15 -year period. $P$ values of $<0.05$ were considered statistically significant. Results. Of the 3,163 cases of posttraumatic endophthalmitis, 1,003 culture-positive isolates were identified. Among these, there were 848 ( $84.5 \%$ ) Grampositive isolates, 109 (10.9\%) Gram-negative isolates, and 46 (4.6\%) fungal isolates. The most common isolates were Staphylococcal species. There was a significant increase in the percentage of fungal isolates over the 15-year period $(P=0.02)$. Gram-positive organisms showed the greatest level of susceptibility to vancomycin $(99.6 \%)$. The susceptibilities of the 109 Gram-negative isolated organisms were as follows: levofloxacin (95.8\%), meropenem (95.7\%), ciprofloxacin (93.5\%), tobramycin $(90.8 \%)$, imipenem $(88.9 \%)$, trimethoprim-sulfamethoxazole (TMP-SMX) $(87.7 \%)$, ertapenem (80\%), and ceftazidime (79.1\%). The susceptibility of Gram-positive organisms to several antibiotics, including levofloxacin $(P=0.004)$, ciprofloxacin $(P<0.001)$, and chloramphenicol $(P=0.001)$ decreased over time, whereas the susceptibility to TMP-SMX increased over time $(P<0.001)$. The susceptibility of Gram-negative bacilli to ceftazidime decreased over time $(P=0.03)$. Conclusions. Over the 15 -year study period, most isolates were Gram-positive cocci, especially coagulasenegative staphylococci (CNS). Vancomycin seemed to be the most effective antibiotic for Gram-positive bacteria. Gramnegative bacteria appeared to be most susceptible to fluoroquinolones. A number of antibiotics showed an increasing trend of microbial resistance.
\end{abstract}

\section{Introduction}

Endophthalmitis is a devastating clinical condition that can lead to severe visual loss. [1-4] Previous research reported that the incidence rate of infectious endophthalmitis following intraocular foreign body (IOFB) injuries ranged from $6.9-30 \%$ [5]. IOFB injuries were reported in $43 \%$ of posttraumatic endophthalmitis cases [6]. A number of studies showed that the specific characteristics of the IOFB, microorganisms, and time between the injury and treatment were associated with an increased risk of endophthalmitis following penetrating trauma [7-9]. An understanding of the spectrum of pathogens and their antibiotic susceptibilities is essential to guide first-line empirical treatment. Information on the microbial spectra and antibiotic susceptibilities of microbes involved in endophthalmitis following IOFB injuries is limited, with no studies on this issue in East China.

The purpose of this study was to investigate the spectrum of pathogens in culture-proven endophthalmitis and related antibiotic susceptibilities at a tertiary hospital in Shanghai. 
TABLE 1: Microbiological spectrum of isolated organisms from 2004 to 2019 .

\begin{tabular}{lcc}
\hline Organisms & $N$ & Total (\%) \\
\hline Gram positive & 848 & 84.5 \\
Staphylococcus epidermidis & 303 & 30.2 \\
Staphylococcus aureus & 113 & 11.3 \\
Other CNS & 259 & 25.8 \\
Streptococcus species \& amp & 36 & 3.6 \\
Corynebacterium species & 27 & 2.7 \\
Bacillus species & 97 & 9.7 \\
Other Gram-positive bacteria & 13 & 1.3 \\
Gram negative & 109 & 10.9 \\
Enterobacteriaceae* & 51 & 5.1 \\
Gram-negative** & 23 & 2.3 \\
Gram-negative bacteria\# & 35 & 3.5 \\
Fungi & 46 & 4.6 \\
Total & 1003 & 100 \\
\hline
\end{tabular}

\& refres to alpha hemolytic streptococcus and beta hemolytic streptococcus Bacillus cereus and Bacillus subtilis * K. pneumoniae, E. coli, Serratia sp., Enterobacter agglomerans, Proteus sp., and Enterobacter sp. ${ }^{* *}$ Acinetobacter sp., Haemophilus sp., Moraxella sp., and Neisseria sp. \#Flavobacterium breve, Pseudomonas maltophilia, Vibrio flurialis, Flavobacterium indologenes, Pseudomonas stutzeri, Pseudomonas oryzihabitans, and Pseudomonas aeruginosa

\section{Materials and Methods}

This was a retrospective review of 3,163 patients admitted to the Eye, Ear, Nose, and Throat (ENT) Hospital, Shanghai Medical College from 1 July 2004 to 31 July 2019. The study was performed in compliance with the principles of the Declaration of Helsinki and was approved by the ethics committee of the Eye and ENT Hospital (no. 2015011). Informed consent was not required as the data were obtained from patients' clinical records in the medical database of the Eye and ENT Hospital.

The ocular examination included the collection of IOFB, aqueous, and vitreous samples. The samples were inoculated onto blood agar, chocolate agar, and brain heart infusion broth and incubated at $37^{\circ} \mathrm{C}$. For fungal cultures, the specimens were inoculated on Sabouraud's dextrose agar and incubated at $25^{\circ} \mathrm{C}$. Bacterial identification was performed using a MicroScan AutoScan system (Dade MicroScan Inc., Sacramento, CA, USA). Antibiotic susceptibility testing was performed using a MicroScan AutoScan system (Dade MicroScan Inc., Sacramento, CA, USA) or the $E$ test (bioMérieux, France). Antibiotic susceptibility was determined according to the guidelines of the Clinical and Laboratory Standards Institute.

All analyses were performed using SPSS, version 21.0 for Windows (SPSS Inc., Chicago, IL, USA). In the statistical analyses, Chi-squared tests were used to detect trends. $P$ values $<0.05$ were considered significant.

\section{Results}

Over the 15-year study period, 1,003 culture-positive isolates were identified among the 3,163 cases of IOFB injuries. The mean age of the patients was $37 \pm 15 y$, and $90.6 \%$ of the patients were males.

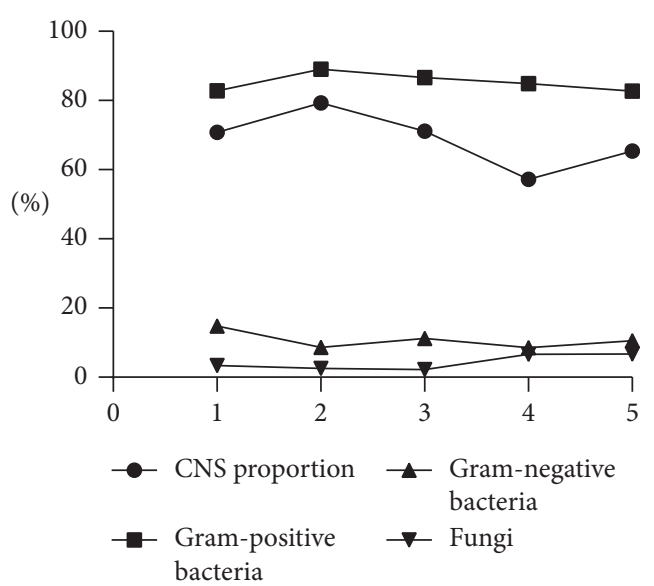

FIgURE 1: Time trends of different isolated organisms from 2004 to 2019. 1, July 2004 to June 2007; 2, July 2007 to June 2010; 3, July 2010 to June 2013; 4, July 2013 to June 2016; 5, July 2016 to July 2019.

3.1. Microbiological Spectrum. In terms of the microbiological spectrum, there were 848 (84.5\%) Gram-positive isolates, 109 (10.9\%) Gram-negative isolates, and 46 (4.6\%) fungal isolates. The most common organisms were Staphylococcal species, with Staphylococcus epidermidis in 303 cases, other CNS in 259 cases, and Staphylococcus aureus in 113 cases. Table 1 presents a detailed overview of the microbial isolates.

3.2. Changes in the Microbiological Spectrum. As shown in Figure 1, the percentage of fungal isolates increased significantly occurred over the 15 -year period $(P=0.02)$. Trends for other isolates were not statistically significant. Trends in the percentage of Gram-positive bacteria $(P=0.797)$ and Gram-negative bacteria $(P=0.586)$ and the proportion of CNS among Gram-positive bacteria $(P=0.203)$ did not reach statistical significance.

3.3. Antibiotic Susceptibilities of the Cultured Gram-Positive Organisms. Table 2 provides information on the antibiotic susceptibilities of the Gram-positive organisms identified. The majority of Gram-positive organisms showed susceptibility to vancomycin (99.6\%), followed by moxifloxacin (90.9\%), levofloxacin $(82.3 \%)$, ofloxacin $(78.1 \%)$, chloramphenicol (78.0\%), and ciprofloxacin (64.7\%). Staphylococcal species were highly susceptible to vancomycin (100\%).

\subsection{Antibiotic Susceptibilities of the Cultured Gram-Negative} Organisms. The antibiotic susceptibilities of the 109 Gramnegative isolates were as follows: levofloxacin (95.8\%), meropenem (95.7\%), gentamicin (95.7\%), amikacin (94.6\%), ciprofloxacin (93.5\%), tobramycin (90.8\%), imipenem (88.9\%), TMP-SMX (87.7\%), ertapenem (80\%), and ceftazidime $(79.1 \%)$ (Table 3$)$.

3.5. Time Trends in Bacterial Susceptibilities to Antibiotics. Chi-squared tests were performed to examine trends in susceptibilities. The results are shown in Figure 2. The susceptibility 
Table 2: Antibiotic susceptibilities of Gram-positive organisms from 2004 to 2019.

\begin{tabular}{lcc}
\hline Antibiotic & Number of susceptible/total number tested & Percent susceptible \\
\hline Vancomycin & $775 / 778$ & 99.6 \\
Penicillins/beta lactams & & \\
$\quad$ Penicillin G & $256 / 735$ & 34.8 \\
$\quad$ Methicillin/oxacillin & $268 / 680$ & 39.4 \\
Fluoroquinolones & & 64.7 \\
$\quad$ Ciprofloxacin & $493 / 763$ & 78.1 \\
Ofloxacin & $364 / 466$ & 82.3 \\
Levofloxacin & $469 / 570$ & 90.9 \\
Moxifloxacin & $140 / 154$ & 32.8 \\
Others & & 78.0 \\
Erythromycin & $243 / 740$ & 60.3 \\
Chloramphenicol & $478 / 613$ & 66.9 \\
TMP-SMX & $248 / 411$ & $255 / 381$ \\
Tobramycin & & \\
\hline
\end{tabular}

Table 3: Antibiotic susceptibilities of Gram-negative organisms from 2004 to 2019.

\begin{tabular}{lcc}
\hline Antibiotic & Number of susceptible/total number tested & Percent susceptible \\
\hline Penicillins/beta lactams & & \\
$\quad$ Ampicillin & $19 / 58$ & 32.8 \\
Cephems & & $26 / 36$ \\
$\quad$ Cefuroxime & $54 / 79$ & 72.2 \\
Ceftriaxone & $72 / 91$ & 68.4 \\
Ceftazidime & & 79.1 \\
Aminoglycosides & $89 / 93$ & 95.7 \\
Gentamicin & $69 / 76$ & 90.8 \\
Tobramycin & $87 / 92$ & 94.6 \\
Amikacin & & \\
Fluoroquinolones & $86 / 92$ & 93.5 \\
Ciprofloxacin & $68 / 71$ & 95.8 \\
Levofloxacin & & 88.9 \\
Others & $64 / 72$ & 95.7 \\
Imipenem & $45 / 47$ & 80.0 \\
Meropenem & $24 / 30$ & 87.7 \\
Ertapenem & $64 / 73$ & \\
TMP-SMX & & \\
\hline
\end{tabular}

of Gram-positive organisms to several antibiotics, including levofloxacin $(P=0.004)$, ciprofloxacin $(P<0.001)$, and chloramphenicol $(P=0.001)$ decreased over time, whereas the susceptibility to TMP-SMX $(P<0.001)$ increased over time. The susceptibility of Gram-negative bacilli to ceftazidime $(P=0.03)$ decreased over time. Trends in susceptibilities for other organisms were not statistically significant (Table 4).

\section{Discussion}

Many studies have described series of posttraumatic endophthalmitis and the distribution of isolates worldwide $[1,6,10,11]$. These studies showed that the susceptibilities of microbiological isolates and infectious agents to particular antibiotics varied over time and differed according to regional variability, population, and ethnicity [12-16]. The findings of these studies point to the importance of regular periodic reviews of local susceptibilities to ensure that the most appropriate antibiotics are used to treat infections.

Previous research reported positive cultures in $6.9-43 \%$ of posttraumatic endophthalmitis cases [3]. Cultures were positive in $31.7 \%$ of eyes in the present series. In terms of the distribution of these isolates, the microbiological spectrum in the present study was generally similar to that found in other reports. [17] CNS were the most common causative pathogen in this study, which agreed with the findings of previous studies $[6,16,18,19]$. In contrast, CNS were involved in $56.0 \%$ of endophthalmitis cases in our study versus $23.1 \%$ in a study conducted in France [20] (Table 1). A previous study reported a high incidence of endophthalmitis caused by Staphylococcal species following open-globe injuries [11]. In these cases, endophthalmitis was likely the result of normal skin flora and contamination of open wounds [18]. Studies also reported that Pseudomonas and Clostridium species caused fulminant endophthalmitis $[21,22]$. Fungal endophthalmitis is less common than bacterial endophthalmitis, which should be suspected in cases of wood or soil contamination in mild and moist climates. The incidence of fungal isolates in our study was lower than that reported in a previous study (4.6\% vs. $16.8 \%)$ [13].

In terms of antibiotic sensitivities and empirical antibiotics, our study confirmed that Gram-positive isolates 


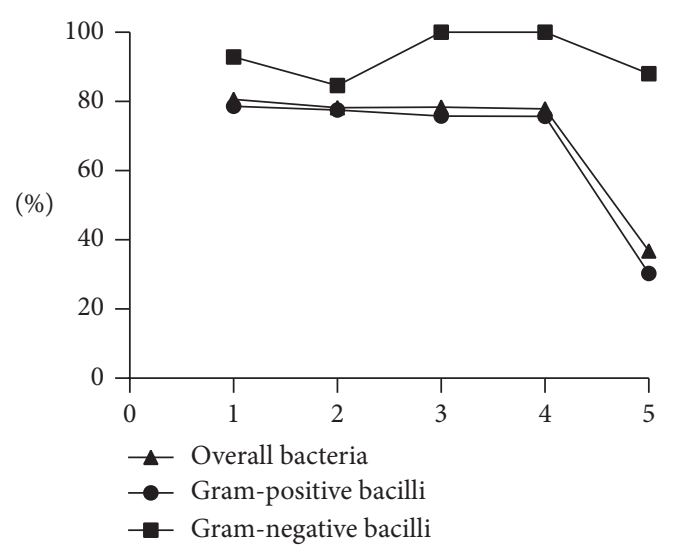

(a)

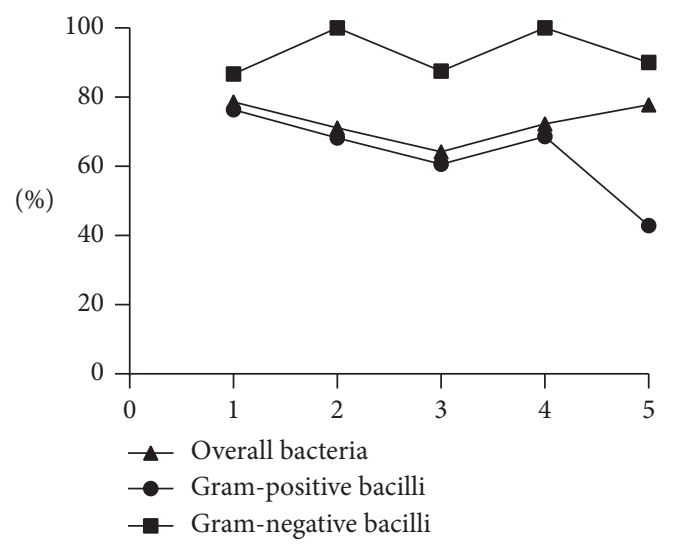

(c)

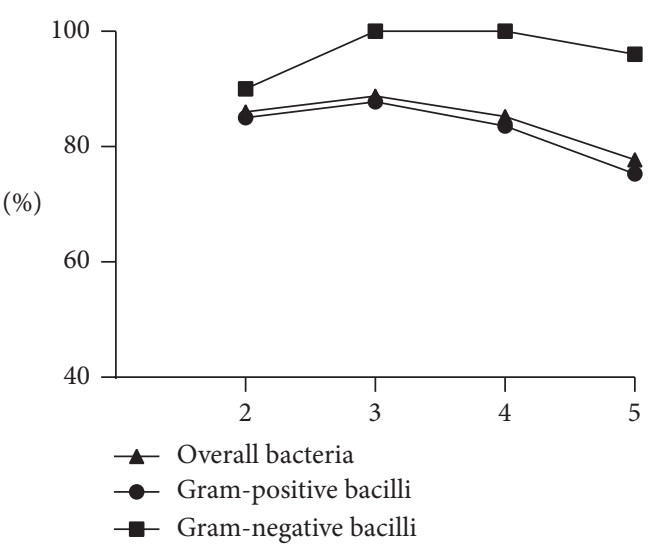

(b)

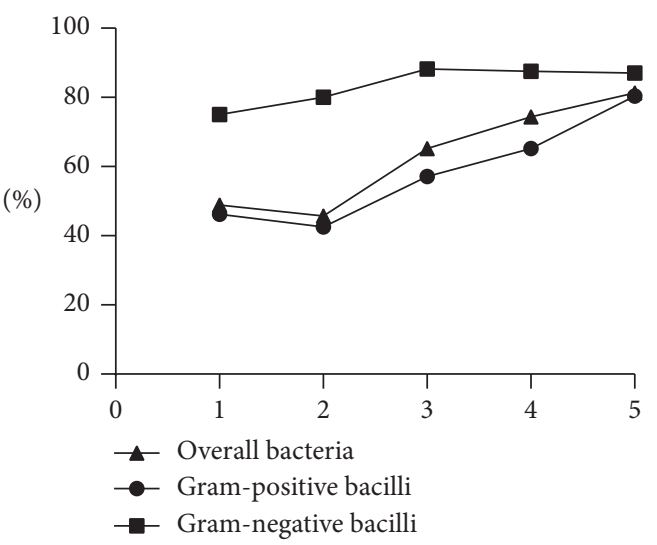

(d)

FIgURE 2: Time trends of bacterial susceptibilities. 1, July 2004 to June 2007; 2, July 2007 to June 2010; 3, July 2010 to June 2013; 4, July 2013 to June 2016; 5, July 2016 to July 2019. Susceptibility testing was unavailable for levofloxacin from July 2004 to July 2007. (a) Ciprofloxacin. (b) Levofloxacin. (c) Tobramycin. (d) TMP-SMX.

Table 4: Antibiotic susceptibilities trends for organisms from 2004 to 2019.

\begin{tabular}{lccc}
\hline$P$ value* & Overall bacteria & Gram-positive bacteria & Gram-negative bacteria \\
\hline Ciprofloxacin & $<0.001$ & $<0.001$ & 0.943 \\
Levofloxacin & 0.005 & 0.004 & 0.726 \\
Tobramycin & 0.629 & 0.173 & 0.767 \\
TMP-SMX & $<0.001$ & $<0.001$ & 0.416 \\
Chloramphenicol & $/$ & 0.001 & $/$ \\
Ceftazidime & $/$ & $/$ & 0.003 \\
\hline
\end{tabular}

${ }^{*} \chi 2$ test for trend. Underlined values mean statistically significant at $P<0.05$.

were generally susceptible to vancomycin (Table 2), which was consistent with the findings of previous reports $[16,23,24]$. The high susceptibility rate of Gram-positive bacteria to vancomycin (99.6\%) supports its continued use as empirical therapy. In previous studies, the sensitivity of Gram-negative isolates to ceftazidime differed according to regional variability, with figures of $91.5 \%, 80.0 \%$, and $77.2 \%$ reported $[10,23,25]$. In our study, $79.1 \%$ of Gram-negative organisms were susceptible to ceftazidime. In addition, the susceptibility of Gram-negative organisms to ceftazidime decreased over time $(P=0.03)$. These findings raise questions around the potential need for changes in empirical therapy, as well as whether intravitreal fluoroquinolone can be a substitute for ceftazidime. Changes in empirical therapy would need to be guided by local microbiological susceptibility patterns. Our series confirmed that the susceptibility of Gram-negative isolates to fluoroquinolones (levofloxacin, 95.8\%; ciprofloxacin, 93.5\%) was greater than their susceptibility to ceftazidime $(79.1 \%)$. Previous studies demonstrated that ciprofloxacin penetrated the blood-ocular barrier, pointing to its use as an intraocular drug [26, 27]. Ocular toxicity appears to be dose-dependent and results from class-effects and specific fluoroquinolone structures, which has been investigated only in animal models [28, 29]. However, the reports regarding the safety of intracameral injection of moxifloxacin has increased [30-34]. Moxifloxacin 
provides superior coverage against Gram-positive organisms, and it also maintains excellent coverage for Gramnegative organisms [35].

Topical fluoroquinolones, which offer broad-spectrum antimicrobial coverage and good ocular penetration, are increasingly used. A recent study reported resistance of endophthalmitis and other ophthalmic isolates to fluoroquinolones [36]. In the present study, levofloxacin and ciprofloxacin exhibited a trend toward increased resistance to Gram-positive bacteria $(P=0.004$ and $P<0.001$, respectively). Increased use of topical fluoroquinolones may lead to resistance in bacterial flora and have a detrimental effect on eye health. Such resistance could partially explain the emergence of fluoroquinolone resistance in ocular microbiology $[37,38]$. In the present study, the susceptibility rates of Gram-negative microorganisms to fluoroquinolones were higher than those of Gram-positive microorganisms, which paralleled the findings of previous research [23].

\section{Conclusion}

The present study has some limitations. It was a retrospective, laboratory study that lacked clinical data. We had access only to microbiological records. In addition, due to technical limitations, anaerobic cultures were not performed. Finally, we could not confirm a correlation between the in vitro susceptibility of microbiological isolates and clinical outcomes.

In conclusion, in this 15-year study, Gram-positive cocci, especially CNS, were the most common organisms isolated in posttraumatic endophthalmitis. There was a significant increase in the percentage of fungal isolates over time. Vancomycin seemed to be the most effective antibiotic for Gram-positive bacteria. Gram-negative bacteria were most susceptible to fluoroquinolones. The susceptibility of the isolates to several antibiotics decreased over time. Antibiotic resistance remains a challenge. Continued surveillance of microbiological isolates provides critical information to guide selection of the most appropriate antibiotics used for empirical management of endophthalmitis.

\section{Data Availability}

The data sets used and/or analyzed during the current study are available from the corresponding author on reasonable request.

\section{Conflicts of Interest}

The authors declare there are no conflicts of interest.

\section{Authors' Contributions}

Wenjun Cao and Xinghuai Sun contributed equally to this work as corresponding authors. Chunhong Liu, Wenjun Cao, and Xinghuai Sun conceived the paper. Chunhong Liu and Jian Ji conducted the data analysis. Chunhong Liu prepared the initial draft of the manuscript. Zhujian Wang and Huiwen Chen equally assisted in data analysis. All authors have read and approved the final manuscript.

\section{Acknowledgments}

This work was supported by the Funds for International Cooperation and Exchange of the National Natural Science Foundation of China (grant no. 81020108017), the National Health and Family Planning Commission, China (grant no. 201302015), and the State Key Program of National Natural Science Foundation of China (grant no. 81430007).

\section{References}

[1] T. P. Banker, A. J. McClellan, B. D. Wilson et al., "Culturepositive endophthalmitis after open globe injuries with and without retained intraocular foreign bodies," Ophthalmic Surgery, Lasers and Imaging Retina, vol. 48, no. 8, pp. 632637, 2017.

[2] P. Garg, A. Roy, and S. Sharma, "Endophthalmitis after cataract surgery," Current Opinion in Ophthalmology, vol. 28, no. 1, pp. 67-72, 2017.

[3] M. L. Durand, "Bacterial and fungal endophthalmitis," Clinical Microbiology Reviews, vol. 30, no. 3, pp. 597-613, 2017.

[4] S. N. Patel, P. P. Storey, M. Pancholy et al., "Changes in management based on vitreous culture in endophthalmitis after intravitreal anti-vascular endothelial growth factor injection," American Journal of Ophthalmology, vol. 207, pp. 224-231, 2019.

[5] C. M. Cebulla and H. W. Flynn Jr., "Endophthalmitis after open globe injuries," American Journal of Ophthalmology, vol. 147, no. 4, pp. 567-568, 2009.

[6] A. M. Al-Omran, E. B. Abboud, and A. M. Abu El-Asrar, "Microbiologic spectrum and visual outcome of posttraumatic endophthalmitis," Retina, vol. 27, no. 2, pp. 236-242, 2007.

[7] R. Essex, Q. Yi, P. Charles, and P. Allen, "Post-traumatic endophthalmitis," Ophthalmology, vol. 111, no. 11, pp. 2015-2022, 2004.

[8] J. B. Jonas, H. L. J. Knorr, and W. M. Budde, "Prognostic factors in ocular injuries caused by intraocular or retrobulbar foreign bodies," Ophthalmology, vol. 107, no. 5, pp. 823-828, 2000.

[9] F. Duan, Z. Yuan, J. Liao, Y. Zheng, Y. Yang, and X. Lin, "Incidence and risk factors of intraocular foreign body-related endophthalmitis in Southern China," Journal of Ophthalmology, vol. 2018, pp. 1-5, 2018.

[10] A. Jindal, A. Pathengay, K. Mithal et al., "Endophthalmitis after open globe injuries: changes in microbiological spectrum and isolate susceptibility patterns over 14 years," Journal of Ophthalmic Inflammation and Infection, vol. 4, no. 1, p. 5, 2014.

[11] H. S. Al-Mezaine, E. A. Osman, D. Kangave, and A. M. Abu El-Asrar, "Risk factors for culture-positive endophthalmitis after repair of open globe injuries," European Journal of Ophthalmology, vol. 20, no. 1, pp. 201-208, 2010.

[12] J.-H. Cheng, Y.-H. Chang, C.-L. Chen, Y.-H. Chen, D.-W. Lu, and J.-T. Chen, "Acute endophthalmitis after cataract surgery at a referral centre in Northern Taiwan: review of the causative organisms, antibiotic susceptibility, and clinical features," Eye, vol. 24, no. 8, pp. 1359-1365, 2010.

[13] F. M. Recchia, B. G. Busbee, R. B. Pearlman, C. A. CarvalhoRecchia, and A. C. Ho, "Changing trends in the microbiologic 
aspects of postcataract endophthalmitis," Archives of Ophthalmology, vol. 123, no. 3, pp. 341-346, 2005.

[14] X. Sun, S. Deng, R. Li et al., "Distribution and shifting trends of bacterial keratitis in north China (1989-98)," British Journal of Ophthalmology, vol. 88, no. 2, pp. 165-166, 2004.

[15] J. Hong, J. Xu, J. Hua, and X. Sun, "Bacterial keratitis in Shanghai," Ophthalmology, vol. 120, no. 3, p. 647, 2013.

[16] C. Liu, J. Ji, S. Li et al., "Microbiological isolates and antibiotic susceptibilities: a 10-year review of culture-proven endophthalmitis cases," Current Eye Research, vol. 42, no. 3, pp. 443-447, 2017.

[17] C. Long, B. Liu, C. Xu, Y. Jing, Z. Yuan, and X. Lin, "Causative organisms of post-traumatic endophthalmitis: a 20 -year retrospective study," BMC Ophthalmology, vol. 14, no. 1, p. 34, 2014.

[18] S. Chhabra, D. Y. Kunimoto, L. Kazi et al., "Endophthalmitis after open globe injury: microbiologic spectrum and susceptibilities of isolates," American Journal of Ophthalmology, vol. 142, no. 5, pp. 852-854, 2006.

[19] A. M. Schimel, D. Miller, and H. W. Flynn Jr., "Endophthalmitis isolates and antibiotic susceptibilities: a 10-year review of culture-proven cases," American Journal of Ophthalmology, vol. 156, no. 1, pp. 50-52, 2013.

[20] P.-L. Cornut, E. B. Youssef, A. Bron et al., "A multicentre prospective study of post-traumatic endophthalmitis," Acta Ophthalmologica, vol. 91, no. 5, pp. 475-482, 2013.

[21] R. Ramakrishnan, M. J. Bharathi, C. Shivkumar et al., "Microbiological profile of culture-proven cases of exogenous and endogenous endophthalmitis: a 10-year retrospective study," Eye, vol. 23, no. 4, pp. 945-956, 2009.

[22] M. N. Iyer, G. Kranias, and M. E. Daun, "Post-traumatic endophthalmitis involving Clostridium tetani and Bacillus spp," American Journal of Ophthalmology, vol. 132, no. 1, pp. 116-117, 2001.

[23] R. C. Gentile, S. Shukla, M. Shah et al., "Microbiological spectrum and antibiotic sensitivity in endophthalmitis," Ophthalmology, vol. 121, no. 8, pp. 1634-1642, 2014.

[24] V. Vedantham, "Ciprofloxacin in endophthalmitis: an emerging alternative," American Journal of Ophthalmology, vol. 137, no. 6, pp. 1167-1168, 2004.

[25] M. S. Benz, I. U. Scott, H. W. Flynn Jr., N. Unonius, and D. Miller, "Endophthalmitis isolates and antibiotic sensitivities: a 6-year review of culture-proven cases," American Journal of Ophthalmology, vol. 137, no. 1, pp. 38-42, 2004.

[26] F. M. Penha, E. B. Rodrigues, M. Maia et al., "Retinal and ocular toxicity in ocular application of drugs and chemicalsPart II: retinal toxicity of current and new drugs," Ophthalmic Research, vol. 44, no. 4, pp. 205-224, 2010.

[27] S. M. Hariprasad, G. K. Shah, W. F. Mieler et al., "Vitreous and aqueous penetration of orally administered moxifloxacin in humans," Archives of Ophthalmology, vol. 124, no. 2, pp. 178-182, 2006.

[28] A. M. Thompson, "Ocular toxicity of fluoroquinolones," Clinical \& Experimental Ophthalmology, vol. 35, no. 6, pp. 566-577, 2007.

[29] H. Gao, M. E. Pennesi, X. Qiao et al., "Intravitreal moxifloxacin: retinal safety study with electroretinography and histopathology in animal models," Investigative Opthalmology \& Visual Science, vol. 47, no. 4, pp. 1606-1611, 2006.

[30] K. Matsuura, T. Miyoshi, C. Suto, J. Akura, and Y. Inoue, "Efficacy and safety of prophylactic intracameral moxifloxacin injection in Japan," Journal of Cataract \& Refractive Surgery, vol. 39, no. 11, pp. 1702-1706, 2013.

[31] R. C. Bowen, A. X. Zhou, S. Bondalapati et al., "Comparative analysis of the safety and efficacy of intracameral cefuroxime, moxifloxacin and vancomycin at the end of cataract surgery: a meta-analysis," British Journal of Ophthalmology, vol. 102, no. 9, pp. 1268-1276, 2018.

[32] A. Haripriya, D. F. Chang, and R. D. Ravindran, "Endophthalmitis reduction with intracameral moxifloxacin prophylaxis," Ophthalmology, vol. 124, no. 6, pp. 768-775, 2017.

[33] V. Galvis, A. Tello, M. A. Sanchez, and P. A. Camacho, "Cohort study of intracameral moxifloxacin in postoperative endophthalmitis prophylaxis," Ophthalmology and Eye Diseases, vol. 6, pp. 1-4, 2014.

[34] S. S. Lane, R. H. Osher, S. Masket, and S. Belani, "Evaluation of the safety of prophylactic intracameral moxifloxacin in cataract surgery," Journal of Cataract \& Refractive Surgery, vol. 34, no. 9, pp. 1451-1459, 2008.

[35] J. M. Blondeau, R. Laskowski, J. Bjarnason, and C. Stewart, "Comparative in vitro activity of gatifloxacin, grepafloxacin, levofloxacin, moxifloxacin and trovafloxacin against 4151 Gram-negative and Gram-positive organisms," International Journal of Antimicrobial Agents, vol. 14, no. 1, pp. 45-50, 2000.

[36] E. Milder, J. Vander, C. Shah, and S. Garg, "Changes in antibiotic resistance patterns of conjunctival flora due to repeated use of topical antibiotics after intravitreal injection," Ophthalmology, vol. 119, no. 7, pp. 1420-1424, 2012.

[37] R. E. Fintelmann, E. N. Hoskins, T. M. Lietman et al., "Topical fluoroquinolone use as a risk factor for in vitro fluoroquinolone resistance in ocular cultures," Archives of Ophthalmology, vol. 129, no. 4, pp. 399-402, 2011.

[38] S. H. Park, J. A. Lim, J. S. Choi, K. A. Kim, and C. K. Joo, "The resistance patterns of normal ocular bacterial flora to $4 \mathrm{flu}$ oroquinolone antibiotics," Cornea, vol. 28, no. 1, pp. 68-72, 2009. 